\title{
3D Reconstruction of the Optic Nerve Head of a Phantom Eye from Images Obtained using a Slit Lamp Fitted with Low Cost Add-Ons
}

\author{
Ian Coghill, Student Member, IEEE-EMBS, Kirsty C. Jordan, Richard A. Black, Iain A.T. Livingstone \\ and Mario E. Giardini, Member, IEEE-EMBS
}

\begin{abstract}
Early detection and treatment are key in limiting vision loss from glaucoma, the second leading cause of blindness worldwide. Morphological alteration of the optic nerve head (ONH), detectable early in the condition, is a key clinical indicator. The mainstay for evaluation in clinics is the subjective assessment of stereoscopic ONH images. If quantitative diagnostic devices, which extract 3D information and use this to make an objective assessment, could be made affordable, it could mean greater diagnostic capability in primary/community care. A potentially cost-effective solution is to extract, using computer stereo vision, 3D information from stereo images obtained through a slit lamp, a mainstay of eye diagnostics, present in practically all ophthalmology and optometry practices. This work shows 3D ONH reconstruction in an eye phantom through a common slit lamp fitted with low cost cameras. Quantitative reconstructions, in close agreement with ground truths, were obtained.
\end{abstract}

\section{INTRODUCTION}

The second leading cause of blindness worldwide, glaucoma [1], is a progressive optic neuropathy characterized by structural change of the optic nerve head (ONH) and irreversible vision loss [2]. Early detection and prompt treatment to slow its progression are key in preventing or limiting vision loss. As an enlargement of the cup of the optical nerve head $(\mathrm{ONH})$, the point where the optic nerve meets the retina, clearly visible when imaging the back of the eye, can begin to occur before visual loss can be detected [3], it is a useful indicator in early detection. It is also a useful indicator to monitor the effectiveness of treatment [4].

The mainstay in clinics for evaluating the $\mathrm{ONH}$ for glaucomatous structural change is the subjective assessment of stereoscopic ONH images [5]. Quantitative imaging devices that can extract the topography of the $\mathrm{ONH}$ and, based on this, make an objective assessment have been shown to have higher diagnostic accuracy than stereo image based $\mathrm{ONH}$ examinations by general ophthalmologists but not glaucoma specialists [6]. In community settings, both in high- and in low-income settings, when specialist healthcare operators cannot be present [6], [7], these devices could be advantageous. As the high costs of these devices [6] is likely prohibitive, a number of groups have been working on a potentially more cost-effective alternative, in which the topography of the $\mathrm{ONH}$ is estimated, using computer stereo vision, from stereo images of the $\mathrm{ONH}$ captured through a

*Research supported by the UK EPSRC (Grant Ref. EP/L015595/1).

I. Coghill, K. C. Jordan, R. A. Black and M.E. Giardini are with the Department of Biomedical Engineering, University of Strathclyde, Glasgow G1 1XQ, UK (e-mail: ian.coghill@strath.ac.uk).

I. A.T. Livingstone is with NHS Forth Valley, Falkirk Community Hospital, Falkirk FK1 5QE, UK (email: iain.livingstone@nhs.net). fundus camera [8]-[12]. Slit lamp biomicroscopes, on the other hand, are universally available in optometry practices and ophthalmology field clinics. A potentially promising idea, considering the still significant cost of fundus cameras, however, is to reconstruct the topography of the $\mathrm{ONH}$ from images acquired, by indirect ophthalmoscopy, using a slit lamp biomicroscope fitted with low cost cameras on the eyepieces. Quantitative work to assess the feasibility of this idea has not been documented to our knowledge. As a step in this direction, the goal of this work was to demonstrate in an eye phantom the feasibility of generating accurate quantitative $3 \mathrm{D}$ reconstructions of normal and glaucomatous ONH models.

\section{Methodology}

In this work, cameras and a slit lamp lens were rigidly mounted to a slit lamp (Fig. 1) to yield a fixed stereo imaging system. This system was then used to capture stereo image pairs of normal and glaucomatous $\mathrm{ONH}$ models in a phantom eye. These image pairs were used to generate quantitative 3D reconstructions using computer stereo vision before being validated against ground truths generated through professional photogrammetry software.

\section{A. Experimental Setup}

A Zeiss 30 SL-M slit lamp (Carl Zeiss AG, Oberkochen, Germany) was used.

A 3D-printed mount was designed to hold a 90D SuperField $\mathrm{NC}^{\circledR}$ slit lamp lens (Volk Optical, Inc., Mentor, Ohio, US) in front, and on the optical axis, of the objective lens of the slit lamp at a distance such that focus on the $\mathrm{ONH}$ models in the phantom eye could be attained.

A 3D-printed camera mount, comprising a $40 \mathrm{~mm}$ Noctua cooling fan (Noctua, Austria) was secured to the eye pieces of the slit lamp and held two Raspberry Pi NoIR v2.1 Cameras (Raspberry Pi Foundation, Cambridge, UK) which feature 8-megapixel Sony IMX219 CMOS sensors.

All 3D printed parts were generated using the CAD software Rhinoceros 6 (McNeel Europe, Barcelona, Spain), and printed in polylactic acid (PLA, RS Components Ltd., Corby, UK) on the Wanhao Duplicator i3 V2.1 fused deposition modelling 3D printer (Wanhao, Zhejiang, China).

The stock lenses were removed from the cameras and replaced with M12 IR Board Lenses with a focal length of 8 $\mathrm{mm}$ (Hongkong Ansice Industrial Co., Ltd., Hong Kong, China), held in position using 3D-printed mounts. The slit lamp was constantly set to x30 magnification, and the focus of the cameras was fixed at infinity. Each of the cameras were connected to a separate Raspberry Pi 3 Model B 


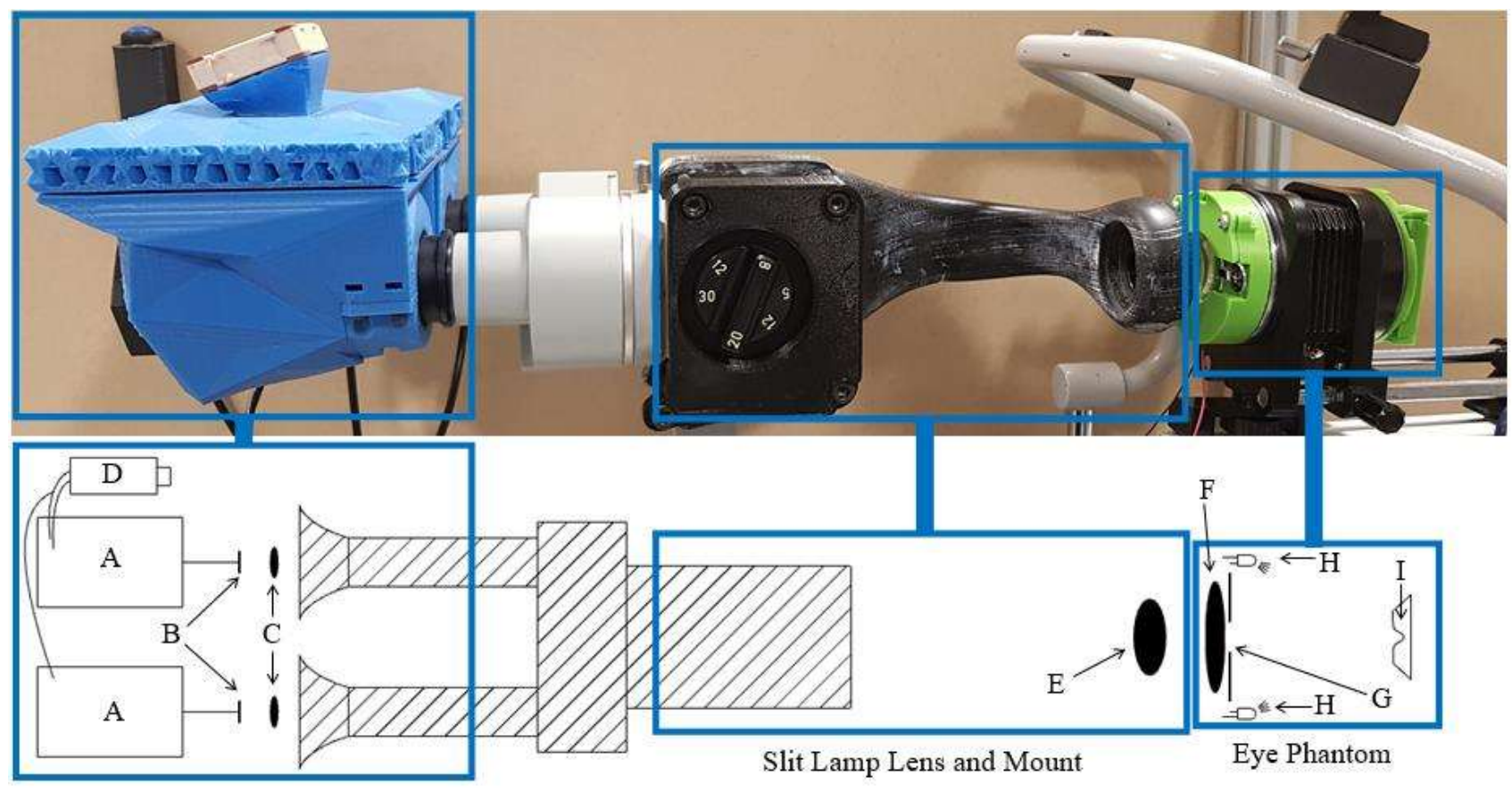

Camera Mount

Figure 1. Slit lamp rigidly mounted with a slit lamp lens and cameras to yield a fixed stereo imaging system which was used to capture stereo images of optic nerve head models in a phantom eye. Hatched area - Zeiss 30 SL-M slit lamp, A - Raspberry Pi 3 Model B computers, B - Raspberry Pi NoIR v2.1 cameras (8-megapixel Sony IMX219 CMOS sensor) with stock lenses removed, C - M12 IR board lenses ( $f=8 \mathrm{~mm}), \mathrm{D}-\mathrm{Push}$ button, E - 90D SuperField $\mathrm{NC}^{\circledR}$ slit lamp lens, F-14 D trial lens, $\mathrm{G}-8 \mathrm{~mm}$ aperture, $\mathrm{H}-2 \mathrm{~mm}$ flat top white LEDs, I - ONH model.

computer (Raspberry Pi Foundation, Cambridge, UK), which were situated inside the camera mount. The two Pi computers transmitted, over $\mathrm{W}-\mathrm{Fi}$, a 720p video stream for aiming, and 8-megapixel uncompressed still snapshots for 3D reconstruction to a laptop running Microsoft Windows 10 (Microsoft Corp., Redmond, Washington, US). A single push button connected to the related IO ports initiated the still image capture command in both Raspberry $\mathrm{Pi}$ computers simultaneously.

An eye phantom - a simplified and scaled up version of a normal human eye - was constructed, comprising a single 14 diopter trial lens to provide refractive power. A 3D-printed mount was used to hold the lens at one end of a macro bellows (Fotga, Shenzhen, China) and an iris (approximately $8 \mathrm{~mm}$ in diameter) just behind this lens. This mount also held two $2 \mathrm{~mm}$ flat top white LEDs (L-13PWC-Z, Kingbright, Issum, Germany) which illuminated the ONH models that were positioned, approximately $71-72 \mathrm{~mm}$ from the lens, by a 3D-printed mount at the other end of the macro bellows. Two different $\mathrm{ONH}$ models were created - one representing a normal and the other a glaucomatous $\mathrm{ONH}$ - based on optical coherence tomography cross-sectional images obtained from the literature. The latter images were uniformly scaled in two-dimensions such that the disc diameter equaled approximately $2 \mathrm{~mm}$. The scaling resulted in a depth of 0.67 $\mathrm{mm}$ and $0.69 \mathrm{~mm}$ from the lowest point of the optic discs' cups to their flatter upper surface for the glaucomatous and normal cases respectively. These values are comparable to HRT and OCT readings seen in the literature [13]. Following scaling, the cross sections were revolved and embedded into the top of truncated square pyramids to yield the 3D designs of the ONH models. The designs of the models were scaled up (uniformly in three-dimensions) appropriately for the scale factor of the eye phantom before being $3 \mathrm{D}$ printed. We note that, by an appropriate size matching between lens focal length and $\mathrm{ONH}$ model, the feature dimensions seen through the slit lamp corresponds to that which is seen in a life-sized human eye. The 3D-printed ONH models were brush painted in titanium white acrylic (Golden Artist Colors, Inc., New York, US) before being partially sprayed with red acrylic paint (WHSmith, Wiltshire, UK) to create a speckled surface texture.

\section{B. Calibration}

The intrinsic and extrinsic calibration parameters of the stereo system (which included the eye phantom lens) were estimated using the Camera Calibration Toolbox for MATLAB ${ }^{\circledR}[14]$. In order to use this, 21 stereo image pairs were acquired of a flat checkboard held in random locations and orientations, at the back of the eye phantom, within the field of view of both cameras. A $6 \times 5$ checkerboard of 2.23 $\mathrm{mm}$ squares, patterned on a film photomask (JD Photo Data, Herts, UK) and glued flat to a standard microscope slide using LOCTITE $\AA 4305$ UV curable glue (Loctite, Dusseldorf, Germany) with a thin white plastic film taped to the other side, was used. It was retroilluminated by two $2 \mathrm{~mm}$ flat top white LEDs (L-13PWC-Z, Kingbright, Issum, Germany).

\section{Quantitative 3D Reconstruction Process}

A $3 \mathrm{D}$ reconstruction program was written in $\mathrm{C}++$ using Visual Studio 2017 Community Edition (Microsoft Corp., Redmond, Washington, US), the OpenCV 3 library [15] and the Point Cloud Library (PCL) [16]. It operated by first undistorting and rectifying the left and right images using the 
estimated stereo camera calibration parameters. This process transformed the image pair such that corresponding points of the left and right images were found on the same horizontal scanlines - transforming the correspondence problem into one which can be solved with a single dimensional search.

Thereafter, a stereo-matching step was employed, to solve the correspondence problem. Firstly, a rectangular region of interest in the left image (template) was selected. The program then searched the right image, in one dimension, for a region of the same size with the best match. This was achieved by sliding the template horizontally over the entire width of the right image, at the same vertical position (y coordinate) as it is in the left image in steps of 1 pixel and calculating the normalized correlation between the template and the patch of the right image beneath it at each step, the patch which corresponded to the highest normalized correlation was regarded the best match. The horizontal disparity between the matching regions was used as the disparity search center (DSC) for the next step.

The initial region of interest selected in the left image was then divided up into a 16 by 16 grid of rectangular blocks, totaling 256 blocks. The program searched the right image for the 256 blocks best matching the 256 blocks in the left image. This was achieved by sliding the blocks horizontally over $\mathrm{a} \pm 30$-pixel range centered around a shift of DSC in the right image, at the same vertical positions (y coordinates) as they are in the left image, in steps of 1 pixel and calculating the normalized correlation between each of the blocks and the patches of the right image beneath them at each step - the patches which corresponded to the highest normalized correlations were regarded as the best matches for the blocks. The disparities between the blocks in the left image and their corresponding best matches in the right image were deemed the pixel-accurate disparities. Following this, the program estimated the disparities with sub-pixel accuracy. To do so, for each block in the left image, a quadratic equation was fitted to the corresponding normalized correlation values for the disparity values in a \pm 3 -pixel range centered around the point of maximum normalized correlation between it and the right image. The terms of the best fit quadratic equation were determined using singular value decomposition. The subpixel accurate disparity was deemed to be the disparity at which the quadratic reached its maximum. Such disparity is inversely proportional to depth, and it was assigned to the center pixel of the block in the left image. The center coordinates of each of the blocks in the left image and their associated disparities were converted into real world coordinates in units of millimeters through reprojection. As the images were initially undistorted (allowing the cameras to be treated as pinhole cameras) reprojection was possible using the perspective transformation matrix (determined using the stereo camera calibration parameters). Disparities were converted to depth using the disparity-to-depth equation [17].

The surface normals were then estimated from the resulting sparse point cloud dataset using the normal estimation feature of the point cloud library. Following this, the normals were sorted such that they were all oriented away from the $\mathrm{ONH}$ model's surface. The point cloud dataset, together with the estimated surface normals, were used to estimate a triangle mesh of the $\mathrm{ONH}$ model's surface using the Poisson based surface reconstruction feature of the point cloud library. The next stage involved generating a dense textured point cloud dataset. This was achieved through the reprojection of each pixel in the template selected in the left image onto the mesh. It involved generating a ray for each pixel then testing each triangle in the mesh, using a raytriangle intersection algorithm, for an intersection. The RGB triplets from the pixels, together with the coordinates of the intersection point of their rays with the mesh, formed the dense textured point cloud dataset (or textured 3D reconstruction).

In order to validate the $3 \mathrm{D}$ reconstructions, ground truth $3 \mathrm{D}$ reconstructions were generated using the commercial photogrammetry software 3DF Zephyr Aerial (3Dflow, Verona, Italy) for comparison. Fifty JPG images were obtained of each $\mathrm{ONH}$ model from different poses, and as close to them as focus would allow, at a resolution of $4032 \times 3024$ pixels using a Samsung Galaxy S8 smartphone (Samsung, Seoul, South Korea). These were input into the software to yield proportionally accurate $3 \mathrm{D}$ reconstructions. These were uniformly scaled in three dimensions to the correct size using the mesh processing software CloudCompare (CloudCompare v2.9.1, 2018). The scaling factor for each was determined from measurements of the diagonal distance of the top surfaces (from corner to corner) of the 3D-printed ONH models (measured by photogrammetry against a ruler scale reference) and of the 3DF Zephyr Aerial generated reconstructions (measured with CloudCompare). CloudCompare was also used to perform the comparison between the obtained $3 \mathrm{D}$ reconstructions and the ground truths. The clouds were roughly aligned manually then finely registered using the fine registration tool. The aligned reconstructions were then sliced to form $0.05 \mathrm{~mm}$ thick cross-sectional profiles, each separated by a distance of $0.5 \mathrm{~mm}$.

\section{RESULTS AND DISCUSSION}

A representative example of stereo image pairs obtained of the glaucomatous ONH model is provided in Fig. 2. The stereo image pair shows that it is feasible to capture narrow field of view, high-quality, stereo images of the ONH model of a phantom eye using this system. Also provided in Fig. 2, are the dense textured $3 \mathrm{D}$ reconstruction and color heightmap textured 3D reconstruction generated of the glaucomatous ONH model (as an example). The dense textured 3D reconstruction is consistent with the glaucomatous $\mathrm{ONH}$ model. It is apparent in the color heightmap textured reconstruction that the cup structure was detected, and that little noise is present. The cross-sectional profiles of aligned $3 \mathrm{D}$ reconstructions and their corresponding ground truths are provided in Fig. 3. It can be seen that the reconstructions match very closely, in terms of shape and scale, with their corresponding ground truths.

As this work was carried out using a phantom eye illuminated from inside, the quality of images when illuminated from outside, as in reality, remains to be determined. It is likely that there will be difficulties resulting from reflections from the slit lamp lens and pre-corneal tear film [18] and potentially lower light intensity due to illumination safety limits. The texture density of the $\mathrm{ONH}$, 
which is arguably much lower than the ONH models used in this work, is also likely to present challenges in the stereo matching step, requiring a tailored stereo matching algorithm, such as the one developed by Bansal and colleagues [10]. Moreover, since the calibration of this system, as described here, is not possible in-vivo, the calibration may need to be carried out on an eye phantom having the appropriate optical characteristics. The accuracy of such calibration protocols will be the subject of further investigation.

\section{CONCLUSION}

This work initially explored the feasibility of the idea of generating accurate quantitative $3 \mathrm{D}$ reconstructions of the

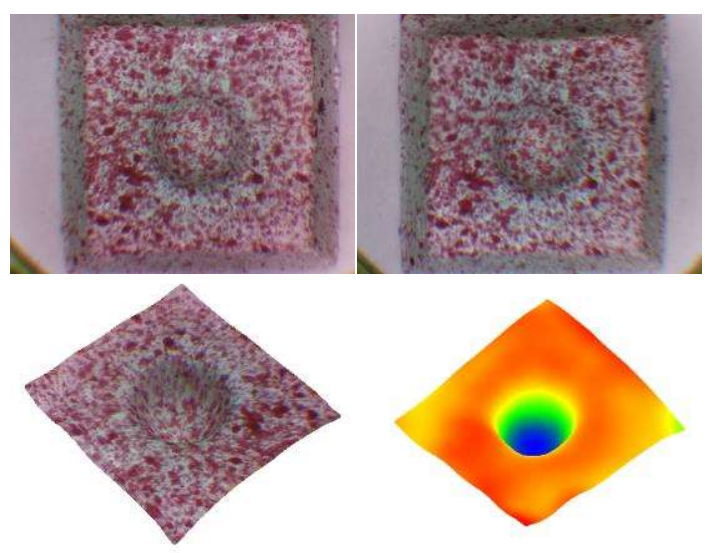

Figure 2. Acquired stereo image pair (top), dense textured 3D reconstruction (bottom left) and color heightmap textured 3D reconstruction (bottom right) of the glaucomatous $\mathrm{ONH}$ model.
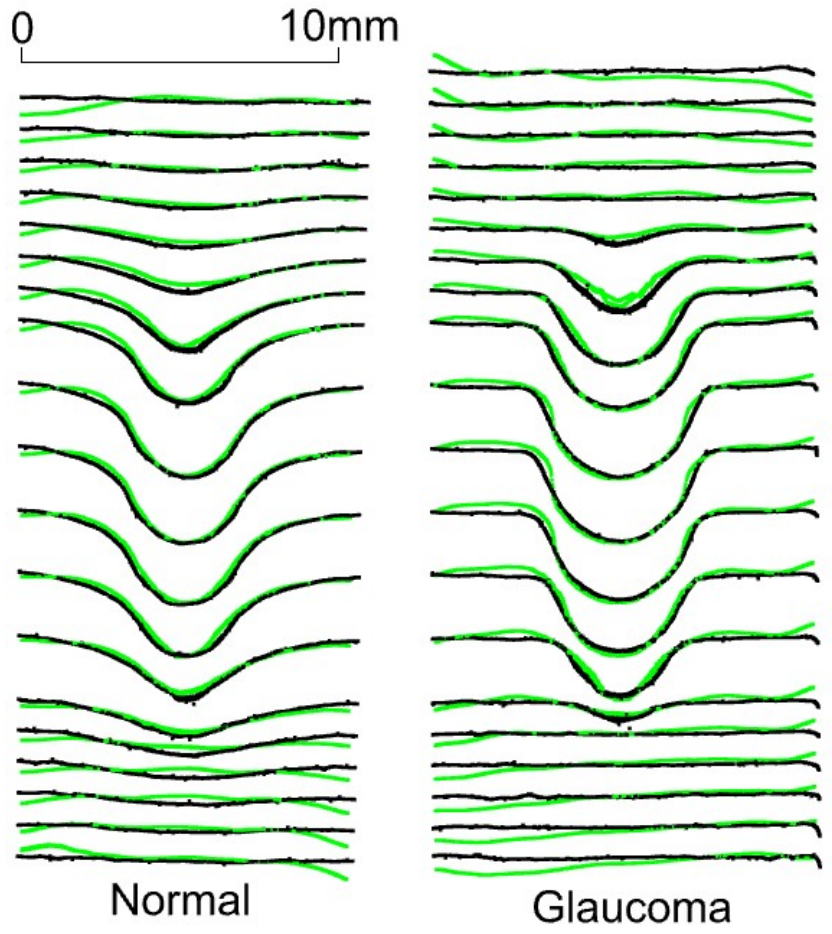

Figure 3. Cross-sectional profiles resulting from extracting $0.05 \mathrm{~mm}$ slices, $0.5 \mathrm{~mm}$ apart, from the aligned 3D reconstructions and their corresponding ground truth. Green $-3 \mathrm{D}$ reconstruction, Black ground truth.
ONH from stereo images obtained through a slit lamp (by indirect ophthalmoscopy) fitted with low cost cameras. Stereo images and resulting quantitative $3 \mathrm{D}$ reconstructions of $\mathrm{ONH}$ models in an eye phantom were obtained. These matched professional photogrammetry software generated ground truths accurately in both shape and scale. The performance of this technique in-vivo is yet to be assessed but the positive results warrant further work to assess the potential of a slit lamp to be used as a quantitative imaging device.

\section{REFERENCES}

[1] S. Kingman, "Glaucoma is second leading cause of blindness globally.," Bull. World Health Organ., vol. 82, no. 11, pp. 887-888, Nov. 2004

[2] H. A. Quigley, “Glaucoma,” Lancet Lond. Engl., vol. 377, no. 9774, pp. 1367-1377, Apr. 2011.

[3] S. J. Mckinnon, "The Value of Stereoscopic Optic Disc Photography," p. 3.

[4] G. Vizzeri, S. M. Kjaergaard, H. L. Rao, and L. M. Zangwill, "Role of imaging in glaucoma diagnosis and follow-up," Indian J. Ophthalmol., vol. 59, no. Suppl1, pp. S59-S68, Jan. 2011.

[5] J. A. Giaconi, S. K. Law, K. Nouri-Mahdavi, A. L. Coleman, and J. Caprioli, Pearls of Glaucoma Management. Springer, 2016.

[6] R. M. Vessani, R. Moritz, L. Batis, R. B. Zagui, S. Bernardoni, and R. Susanna, "Comparison of quantitative imaging devices and subjective optic nerve head assessment by general ophthalmologists to differentiate normal from glaucomatous eyes," J. Glaucoma, vol. 18, no. 3, pp. 253-261, Mar. 2009.

[7] R. Thomas, "Glaucoma in developing countries," Indian J. Ophthalmol., vol. 60, no. 5, pp. 446-450, 2012.

[8] T. Nakagawa et al., "Quantitative depth analysis of optic nerve head using stereo retinal fundus image pair," J. Biomed. Opt., vol. 13, p. 064026, Nov. 2008.

[9] L. Tang, M. K. Garvin, K. Lee, W. L. M. Alward, Y. H. Kwon, and M. D. Abràmoff, "Robust Multiscale Stereo Matching from Fundus Images with Radiometric Differences," IEEE Trans. Pattern Anal. Mach. Intell., vol. 33, no. 11, pp. 2245-2258, Nov. 2011.

[10] M. Bansal, M. Sizintsev, J. Eledath, H. Sawhney, D. J. Pearson, and R. A. Stone, "3D optic disc reconstruction via a global fundus stereo algorithm," Conf. Proc. Annu. Int. Conf. IEEE Eng. Med. Biol. Soc. IEEE Eng. Med. Biol. Soc. Annu. Conf., vol. 2013, pp. 5877-5882, 2013.

[11] E. Corona, S. Mitra, M. Wilson, T. Krile, Y. H. Kwon, and P. Soliz, "Digital stereo image analyzer for generating automated 3-D measures of optic disc deformation in glaucoma," IEEE Trans. Med. Imaging, vol. 21, no. 10, pp. 1244-1253, Oct. 2002.

[12] J. Xu, O. Chutatape, C. Zheng, and P. C. T. Kuan, "Three dimensional optic disc visualisation from stereo images via dual registration and ocular media optical correction," $B r . J$. Ophthalmol., vol. 90, no. 2, pp. 181-185, Feb. 2006.

[13] G. Seymenoğlu, E. Başer, and B. Oztürk, "Comparison of spectraldomain optical coherence tomography and Heidelberg retina tomograph III optic nerve head parameters in glaucoma," Ophthalmol. J. Int. Ophtalmol. Int. J. Ophthalmol. Z. Augenheilkd., vol. 229, no. 2, pp. 101-105, 2013.

[14] J.-Y. Bouguet, Camera Calibration Toolbox for Matlab®. 2018.

[15] G. Bradski, "The OpenCV Library," Dr Dobbs J. Softw. Tools, 2000.

[16] R. B. Rusu and S. Cousins, "3D is here: Point Cloud Library (PCL)," in 2011 IEEE International Conference on Robotics and Automation, 2011, pp. 1-4.

[17] D. Figueira, M. Lopes, R. Ventura, and J. Ruesch, "Towards a Spatial Model for Humanoid Social Robots," 2009, vol. 5816, pp. 287-298.

[18] M. Sheehan and A. Goncharov, "Unwanted reflections during slit lamp assisted binocular indirect ophthalmoscopy," J. Mod. Opt., vol. 58,2011 